\title{
Exploring the properties of lead oxide and tungsten oxide based graphene mixed nanocomposite films
}

\author{
A. Deepak, P. Shankar \\ Saveetha School of Engineering, Saveetha University, Chennai-602105, India \\ deepak.nanozone@gmail.com, principal.sse@saveetha.com
}

DOI 10.17586/2220-8054-2016-7-3-502-505

\begin{abstract}
This work reports the possibility of mixing metal oxides along with graphene and polymer to form Metal Oxide-Graphene-Polymer films. The metal oxides used in this work were lead Oxide $(\mathrm{PbO})$ and tungsten oxide $\left(\mathrm{WO}_{3}\right)$. Polyvinylidene fluoride (PVDF) is the polymer, which is used as matrix in preparation of these metal oxide and graphene mixed nanocomposite films. Metal Oxide-Graphene-PVDF films were developed using a chemical technique called solvent casting. The prepared films were characterized using techniques like FE-SEM, XRD and FT-IR to understand its morphological, crystalline and functional properties. I-V characteristics were analyzed using four-probe techniques. The developed Metal Oxide-Graphene-PVDF films have potential applications in a wide range of fields and particularly for electronic applications. The ability of these films to be used as electronic components in devices was explored.
\end{abstract}

Keywords: graphene, polymer, metal oxide.

Received: 28 January 2016

\section{Introduction}

Graphene is a single layer of carbon atoms peeled from graphite as flakes. Graphene has numerous extraordinary properties which make it exceptionally valuable for different applications [1]. Graphene is preferred compared to carbon Nano tubes, carbon Nano fibers and other carbon materials due to its aspect ratio, high surface area, high tensile strength, good thermal conductivity, good electrical conductivity, transparency and flexibility [2,3]. Graphene is capable of being blended with a wide range of polymer matrices including, epoxy, polystene, polyaniline, nafion, poly (vinyl alcohol), polyurethane, polyvinylidene fluoride, poly (3,4-ethyldioxythiophene), polyethylene terephthalate and polycarborate [4]. Such composites were also shown to exhibit superior properties compared to base polymer materials, as a result of graphene's novel properties. In this present work, graphene along with metal oxides like lead oxide and tungsten oxide were mixed separately with PVDF polymer matrix in order to explore the conductivity behavior of graphene based nanocomposite films before and after mixing with various metal oxides, as previously mentioned.

\section{Materials and methods}

Functionalized graphene were purchased from Ad Nano Technologies with $99 \%$ purity. Lead Oxide and tungsten Oxide were purchased from Sigma Aldrich with $99.4 \%$ purity. Polyvinylidene Fluoride (PVDF) was purchased from Sigma Aldrich with $99 \%$ purity. Dimethylformamide (DMF) of laboratory grade with $98 \%$ purity was purchased from Sigma Aldrich.

FESEM and EDX images were examined using F E I Quanta FEG 200 - High Resolution Scanning Electron Microscope with EDX system. The prepared samples were examined at $50 \mu \mathrm{m}$ range with 1000-2000 x magnification at low vacuum. XRD measurements are carried out using X'pert Pro Powder X-Ray Diffractometer system. The system used the high speed solid-state $X^{\prime}$ celerator as a detector type at the range of $0-100^{\circ}$. FTIR pattern is measured using ALPHA-T FT-IR Spectrometer. The spectral range used for analyzing is 400 to $1000 \mathrm{~cm}^{-1} \mathrm{range}_{\text {. }}$ The spectral resolution used is better than $2 \mathrm{~cm}^{-1}$.

\section{Preparation protocol of nanocomposite films}

\subsection{Preparation of $2 \mathrm{wt} \%$ of graphene-PVDF nanocomposite}

Graphene $(0.04 \mathrm{mg})$ and PVDF $(1.96 \mathrm{mg})$ is mixed with DMF solution and sonicated for 1 hour to get proper mixing in the solution. Obtained graphene-PVDF solution is magnetically stirred for 1 hour by maintaining the temperature between 45 to $65^{\circ} \mathrm{C}$ to get homogenous dispersion in the solution [5]. Finally the solution is kept in hot air oven for 6 hours by keeping constant temperature at $60{ }^{\circ} \mathrm{C}$. 


\subsection{Preparation of $2 \mathrm{wt} \%$ of graphene-PVDF-PbO nanocomposite}

Graphene $(0.04 \mathrm{mg})$, lead Oxide $(0.04 \mathrm{mg})$ and PVDF $(1.92 \mathrm{mg})$ is mixed with a DMF solution and sonicated for 1 hour to get homogenous mixing in the solution. The obtained graphene-PVDF-PbO solution is magnetically stirred for 1 hour by maintaining the temperature between 45 to $65^{\circ} \mathrm{C}$ to get proper dispersion in the solution. Finally, the solution is kept in hot air oven for 6 hours by keeping constant temperature at $60{ }^{\circ} \mathrm{C}$.

\subsection{Preparation of $2 \mathrm{wt} \%$ of graphene-PVDF-WO $\mathrm{W}_{3}$ nanocomposite}

The procedure used is similar to that of graphene-PVDF-PbO nanocomposites. Here lead Oxide is replaced with tungsten Oxide.

\section{Results and discussions}

Figure 1 shows FESEM images of (a) graphene-PVDF, (b) graphene-PVDF-PbO and (c) graphene-PVDF-WO 3 respectively. Superior bonding is noticed which shows the homogenous mixing in the prepared nanocomposites whereas the EDX pattern shows the presence of chemical compounds present in the nanocomposite. EDX images in Fig. 1(d) shows the presence of Carbon and Fluorine in the graphene-PVDF film, in Fig. 1(e) shows the presence of Carbon, Fluorine and Lead in the graphene-PVDF-PbO film and in Fig. 1(f) shows the presence of Carbon, Fluorine and Tungsten in the graphene-PVDF- $\mathrm{WO}_{3}$.
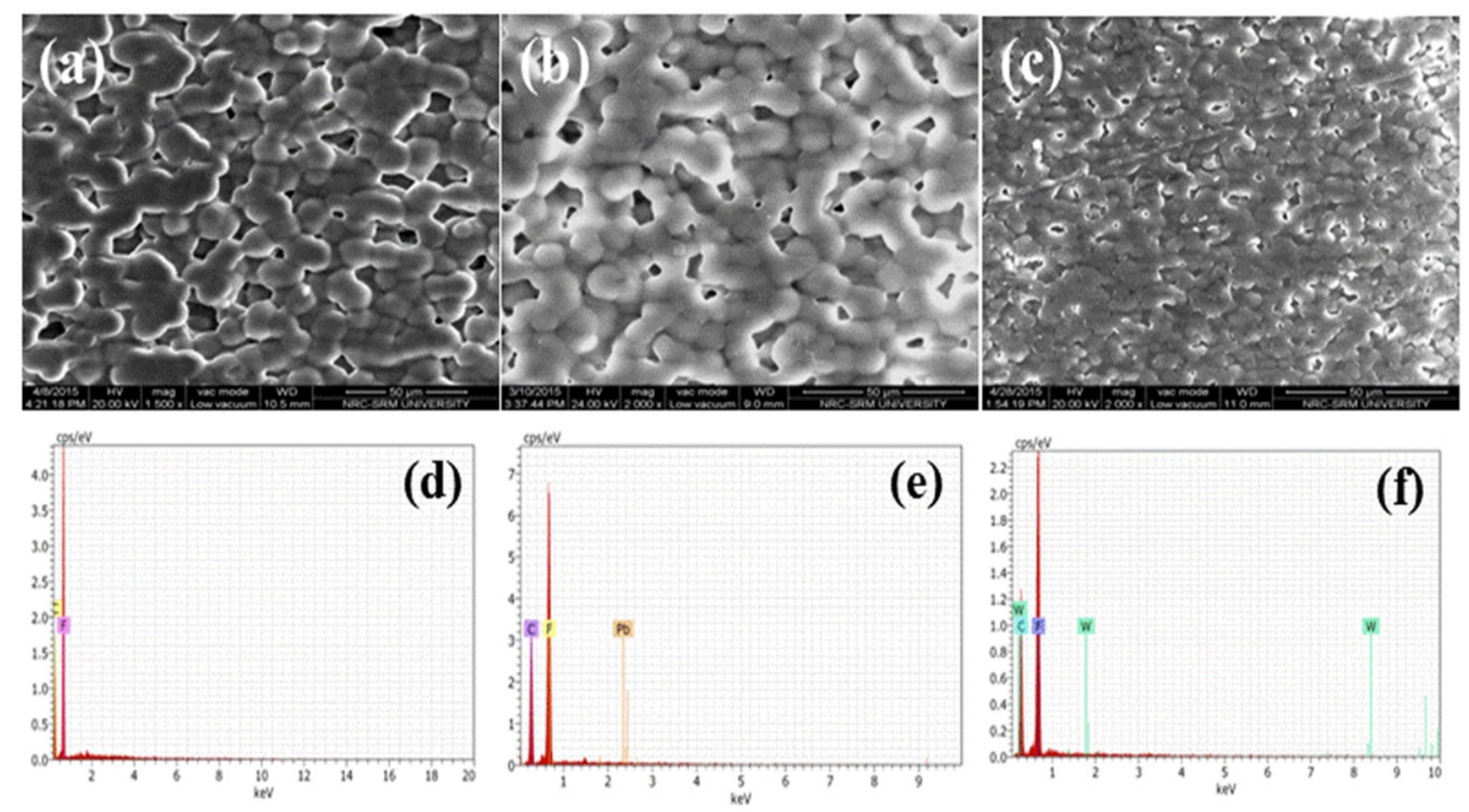

FIG. 1. FESEM images of (a) graphene-PVDF, (b) graphene-PVDF-PbO \& (c) graphene-PVDF$\mathrm{WO}_{3}$ and EDX images of (d) graphene-PVDF, (e) graphene-PVDF-PbO \& (f) graphene-PVDF$\mathrm{WO}_{3}$

Figure 2 shows the XRD pattern of (a) graphene-PVDF, (b) graphene-PVDF-PbO and (c) graphene-PVDF$\mathrm{WO}_{3}$ respectively. Fig. 2(a) shows the $2 \theta$ peaks at $20^{\circ}(110)$ and $40^{\circ}(002)$, which confirms the presence of PVDF and at $26^{\circ}(021)$ as graphene (JCPDS Card No. 00-41-1487). Fig. 2(b) shows the $2 \theta$ peaks at $20^{\circ}$ (110) and $40^{\circ}(002)$ which confirms the presence of PVDF, at $26^{\circ}(021)$ as graphene and at $31^{\circ}(111)$ and $48^{\circ}(220)$ as lead (JCPDS Card No. 00-38-1477) [6]. Fig. 2(c) shows the $2 \theta$ peaks at $20^{\circ}(110)$ and $40^{\circ}(002)$, which confirms the presence of PVDF, at $26^{\circ}(021)$ as graphene and at $18^{\circ}(100), 37.5^{\circ}(021), 45^{\circ}(020)$ and $48^{\circ}(124)$ as Tungsten (JCPDS Card No. 00-43-1035) peak [7]. Fig. 3(a) shows the peaks of graphene-PVDF at $440 \mathrm{~cm}^{-1}$, $485 \mathrm{~cm}^{-1}$ and $510 \mathrm{~cm}^{-1}$. Fig. 3 (b) shows the peaks of graphene-PVDF-PbO at $430 \mathrm{~cm}^{-1}, 470 \mathrm{~cm}^{-1}, 500 \mathrm{~cm}^{-1}$ and $865 \mathrm{~cm}^{-1}$. Fig. 3(c) shows the peaks of graphene-PVDF-WO $\mathrm{WO}_{3}$ at $427 \mathrm{~cm}^{-1}, 465 \mathrm{~cm}^{-1}$ and $860 \mathrm{~cm}^{-1}$. These peaks show the presence of aromatic $\mathrm{C}-\mathrm{C}$ bonds between $\left(400-600 \mathrm{~cm}^{-1}\right)$, $\mathrm{C}-\mathrm{O}$ bonds between $(700-$ $\left.1000 \mathrm{~cm}^{-1}\right)$ and $\mathrm{C}-\mathrm{H}$ bonds between $\left(400-650 \mathrm{~cm}^{-1}\right)$, which is due to the presence of carbon fillers. 


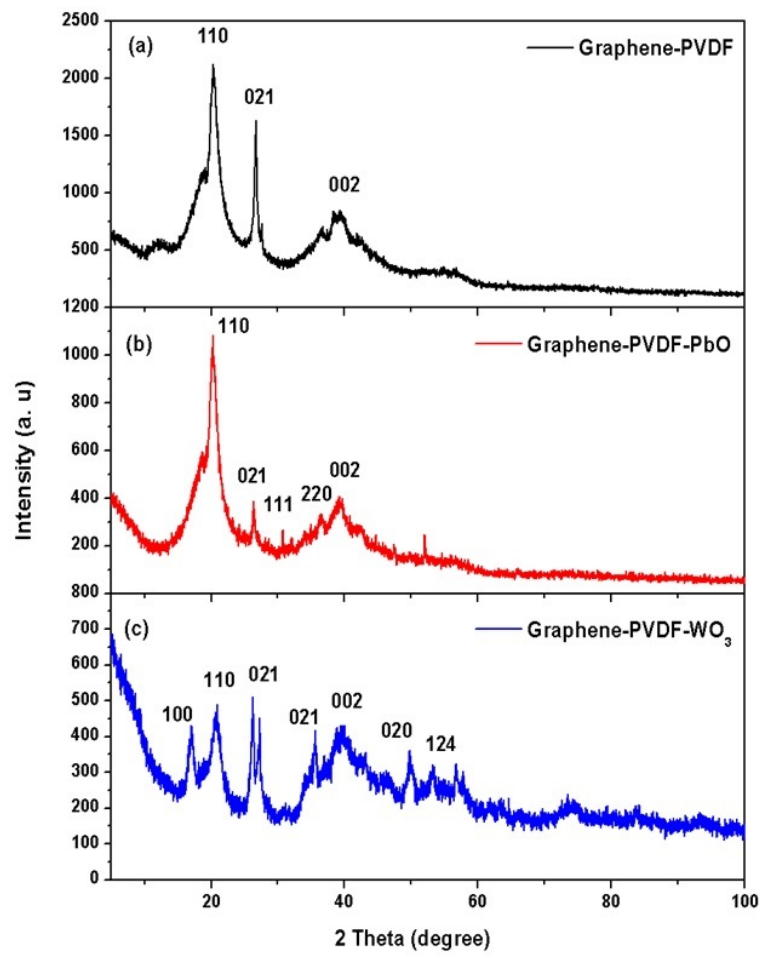

FIG. 2. XRD Pattern of (a) graphene-PVDF, (b) graphenePVDF-PbO and (c) graphene-PVDF$\mathrm{WO}_{3}$

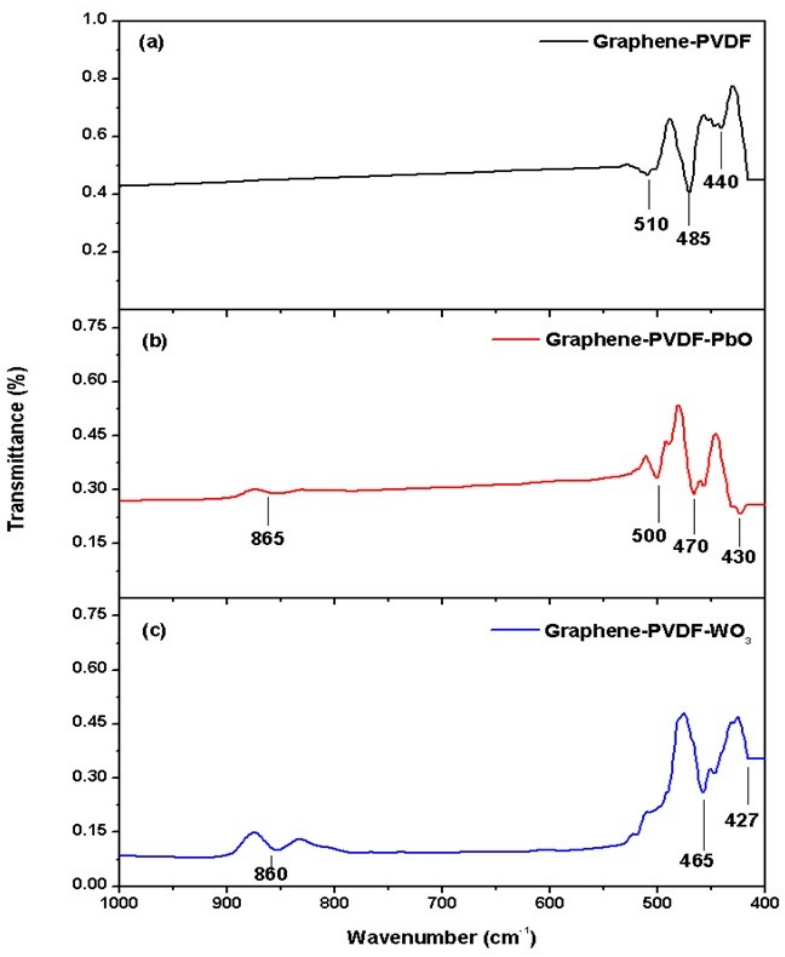

FIG. 3. FTIR Pattern of (a) graphene-PVDF, (b) graphene$\mathrm{PVDF}-\mathrm{PbO}$ and (c) graphene-PVDF$\mathrm{WO}_{3}$

Figure 4 shows the I-V Characteristics of (a) graphene-PVDF, (b) graphene-PVDF-PbO and (c) graphene$\mathrm{PVDF}_{-\mathrm{WO}_{3}}$ were obtained using four probe techniques. Temperature is kept as constant at room temperature and voltage is varied between $0 \mathrm{~V}$ to $1.4 \mathrm{~V}$. Current measurements are carried out across all the three types of nanocomposite films, as shown in Fig. 4. Graphene-PVDF- $\mathrm{WO}_{3}$ shows better conductivity when compared to graphene-PVDF-PbO and graphene-PVDF nanocomposite. This indicates metal oxide graphene based nanocomposite shows better conductivity due to incorporation of metal oxides in graphene based nanocomposites.

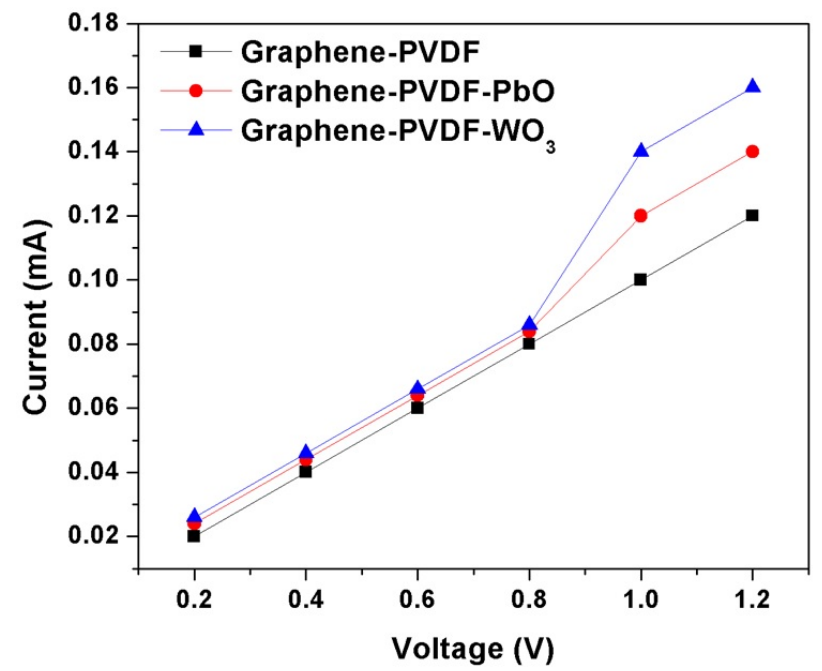

FIG. 4. I-V Characteristics of (a) graphene-PVDF, (b) graphene-PVDF-PbO and (c) graphene$\mathrm{PVDF}-\mathrm{WO}_{3}$ 


\section{Conclusions}

Three different nanocomposite films, namely graphene-PVDF, graphene-PVDF-PbO and graphene-PVDF-WO 3 were prepared. SEM shows the morphological variations in all types of films. EDX shows the presence of elemental compositions such as carbon, fluorine, lead and tungsten of each film individually. XRD spectra shows the peaks of PVDF at $20^{\circ} \& 40^{\circ}$, graphene at $26^{\circ}$, lead at $31^{\circ} \& 48^{\circ}$ and tungsten at $18^{\circ}, 37.5^{\circ}, 45^{\circ} \& 48^{\circ}$. FT-IR spectra show the presence of various functional groups like aromatic $\mathrm{C}-\mathrm{C}$ bonds, $\mathrm{C}-\mathrm{O}$ bonds and $\mathrm{C}-\mathrm{H}$ bonds. Developed nanocomposite films will have utility in flexible electronics in the near future.

\section{References}

[1] Novoselov K.S., Geim A.K., Morozov S.V., Jiang D., Zhang Y., Dubonos S.V., Grigorieva I.V., Firsov A.A. Electric Field Effect in Atomically Thin Carbon Films. Science, 2004, 306, P. 666-669.

[2] Bae S., Kahya O., Sharma B.K., Kwon J., Cho H.C., Ozyilmaz B., Ahn J. Graphene-P(VDF-TrFE) Multilayer Film for Flexible Applications. ACS Nano, 2013, 7, P. 3130-3138.

[3] Shin Keun-Young., Hong Jin-Yong., Jang J. Flexible and transparent grapheme films as acoustic actuator electrodes using inkjet printing. Chemical Communication, 2011, 47, P. 8527-8529.

[4] Mukhopadhyay P., Gupta R.K. Trends and Frontiers in Graphene-Based Polymer. Plastics Engineering, 2011 , P. $32-43$.

[5] Deepak A., Ganesan V., Karthik V., Shankar P. Nanomaterial Based Non- Destructive Evaluation Sensor for Defect Detection and Strain Measurement. Journal of Nanostructured Polymers and Nanocomposites, 2014, 10, P. 5-12.

[6] Arafa H.M. Economically viable route for synthesis of lead oxide $(\alpha$-PbO) nanoparticles with an aim to reduce the manufacturing cost of lead acid batteries. World Journal of Pharmaceutical Sciences, 2014, 2(12), P. 1947-1952.

[7] Hsieh Y-T., Chang L-W., Chang C-C., Wei B-J., Shih H.C. Novel Synthetic Route for Tungsten Oxide Nanobundles with Controllable Morphologies. Recent Trend in Electrochemical Science and Technology, 2012, P. 249-260. 